\title{
Diabetes and Education - Knowledge Level about Diabetes \\ Management in Nurses Student
}

\author{
Požar $\mathrm{H}^{1 *}$, Paro JN ${ }^{2}$ and Simin $\mathrm{D}^{3}$ \\ 1Preschool Teacher and Sport Trainer High school, Serbia \\ ${ }^{2}$ Department of Endocrinology Faculty of Medicine, University of Novi Sad, Serbia \\ ${ }^{3}$ Department of Nursing, Faculty of Medicine, University of Novi Sad, Serbia
}

Research Article

Volume 3 Issue 1

Received Date: January 02, 2019

Published Date: January 25, 2019

DOI: $10.23880 /$ nhij-16000171

*Corresponding author: Hajnalka Požar, Preschool Teacher and Sport Trainer High school, Assistant of Medical Sciences-Health Care, Banijska 67, 24000 Subotica, Serbia, Tel: +381695571980; Email: pozarh@gmail.com

\section{Abstract}

Background: Nurses have an important role in the treatment of people with diabetes. However, the information which is transmitted to patients greatly depends on their knowledge of the subject. The aim of this study was to investigate the level of knowledge about diabetes among professional nurses.

Method: The survey was conducted as a cross-sectional study in which 80 nursing students participated from high school in Subotica. The level of knowledge was evaluated using the Diabetes Knowledge Test. For statistical analysis, we used SPSS statistical program, methods of descriptive statistics.

Results: Respondents have low skills (58.75\%) in nutritional needs. Medium knowledge about controlling blood sugar $(60 \%)$, and intermediate level of knowledge $(63 \%)$ of chronic complications. The total knowledge about diabetes was $64.36 \%$.

Conclusion: The intermediate level of knowledge of diabetes, and low nutrition knowledge, which is the main activity of patient education by nurses, alerts us to possible errors in health educational work.

Keywords: Diabetes; Knowledge; Awareness Information; Nurses; Health Education

\section{Introduction}

Society being burdened by chronic non-communicable diseases is a major public health challenge worldwide, and is one of the main reasons for the use of health care. Although significantly preventable, this disease shows a paradox, although knowledge of these diseases is increasing rapidly and the methods and results of treatment are improving the incidence of these diseases is increasing [1].

Diabetes is one of the most common chronic noncommunicable diseases. The World Health Organization and the International Diabetes Federation estimates that in 2014, 387 million people in the world suffer from diabetes, and that the number of diabetic patients by 2035 will increase to 592 million [2].

The number of newly diagnosed people with type 2 diabetes during 2014 in Serbia was 15.000. The incidence rates of type 2 diabetes per 100.000 inhabitants was 112.3 [3]. According to the estimate of the Institute of Public Health of Serbia, in the Republic of Serbia, diabetes affects approximately 710,000 people or $12.4 \%$ of the adult population, which corresponds to the comparative prevalence of $9.9 \%[4]$. 


\section{Nursing \& Healthcare International Journal}

As a chronic illness effective self-management is critical to the success of the treatments. Diabetic care is primarily self-care, which requires the patient to actively participate in making decisions, setting goals and in the process of daily management. Multiple changes in behavior are needed for diabetic patients to manage their illness effectively and to achieve their desired level of glycemic control. Creating and maintaining behavioral changes is required for the management of diabetes and it requires cooperation between the patient and the provider of health care for the development of a plan. A multidisciplinary team consists of nurses, dietitians, pharmacists and doctors [5].

However, the nurses, as the largest number of medical personnel, and as who spends most of their time with patients, have a major role in educating patients with type 2 diabetes [6]. No matter in what sphere of health care the employed are, in all fields they can encounter the disease, and need to transfer the necessary knowledge to patients. The role of nurses in the education of diabetics is to help patients with self-care and in managing their illness through education and support. However, nurses need to have adequate knowledge and a wide range of professional skills, which include communication, counseling, leadership and a talent for teaching, in order to successfully carry out the training [7].

Therefore, nurses are key treatment providers. Experience and knowledge about diabetes and its management is essential for effective education of patients with diabetes. However, the information which will be transferred by the nurse to the patients depends on their knowledge of this subject, the possession of pedagogical competences, and whether they are capable of supporting patients to initiate and maintain behavior changes associated with the optimal control of diabetes [8].

The aim of this study is to investigate the level of knowledge of students of vocational nurses in relation to diabetes. The main hypothesis is that there is a significant difference among employed and unemployed students because of workplace experience, during which the nurses acquire a certain degree of knowledge and necessary skills.

\section{Materials and Methods}

\section{Participants}

This study was conducted as a cross-sectional study during the school year of $2016 / 17$, with participation 80 students first and second years of medical nurses, at the Preschool Teacher and Sport Trainer High school in Subotica, Serbia. To assess the knowledge a questionnaire was used as a test of knowledge, which includes detailed information on diet, medication, and complications to evaluate the level of knowledge needed for the education for diabetes management. The survey was anonymous and, voluntary, and approved in writing by the director of the institution. By voluntary filling the questionnaires, the participants gave their consent to participate in the examination.

\section{Instrument}

Knowledge about diabetes was measured using a test of knowledge about diabetes (Diabetes Knowledge Test), developed and tested for reliability and validity by scientists at the University of Michigan [9]. The diabetes knowledge test contains 23 multiple-choice points, designed for the evaluation of knowledge about nutrition, testing blood glucose levels, on the management of complications, insulin therapy and self-care activities. The internal consistency of the questionnaire for the skills was measured by Cronbach's alpha coefficient and was adequate (0.78) [10]. The result is determined by dividing the number of correct answers of all questions. Correct answers by the "Diabetes Research and Training Center, University of Michigan" in the results were marked with an asterisk $\left(^{*}\right.$. The results are labeled: $\geq 75 \%$-high, 74$60 \%$ medium and $\leq 59 \%$ - low knowledge about diabetes [11].

\section{Data Analysis}

For statistical analysis we used IBM Corp. SPSS Statistics for Windows, version 20.0, methods of descriptive statistics have been used. The general characteristics of the participants were calculated with the frequency, in percents, with mean values and standard deviations. To test the statistical significance after employment and work experience the Pearson $\chi 2$ test was used.

\section{Results}

\section{General Characteristics of Respondents}

The questionnaire was filled by $16(20 \%)$ men and 64 $(80 \%)$ women. The mean age was $27.33 \pm 7.35$ years. Of these $52(65 \%)$ is employed in a medical institution Table 1 , and $28(35 \%)$ are currently unemployed. The average length of service was $6.50 \pm 6.98$ years. Half $(50 \%)$ of them have stated that during work they regularly perform the health education of diabetics. 


\section{Nursing \& Healthcare International Journal}

\begin{tabular}{|c|c|}
\hline Years of work experience & $\mathbf{\% ( n )}$ \\
\hline $1-5$ years & $60(48)$ \\
\hline 6-10 years & $15(12)$ \\
\hline 11-15 years & $10(8)$ \\
\hline over 16 years & $15(12)$ \\
\hline
\end{tabular}

Table 1: Years of service of the respondents.

\section{Diabetic Nutrition}

To the questions in the field of nutrition therapy in diabetic patients, $58.75 \%$ of the respondents gave the correct answer, which corresponds to low knowledge in this area Table 2. Negative statistically significant correlation was determined. We also found a negative statistical correlation between employment and knowledge about diabetic diet $(\mathrm{p}=0.013)$.

\begin{tabular}{|c|c|c|c|c|c|c|}
\hline & \multirow{2}{*}{ Total \% (n) } & \multicolumn{5}{|c|}{ According to the years of work experience } \\
\hline & & $\mathbf{0}$ & 5-Jan & 10-Jun & 15-Nov & $16-20$ \\
\hline \multicolumn{7}{|c|}{ The diabetes diet is: $p=0.439$} \\
\hline The way most people eat & - & - & - & - & - & - \\
\hline A healthy diet for most people* & $65(52)$ & $83.3(20)$ & $63.6(14)$ & $14.3(2)$ & $50(4)$ & $100(12)$ \\
\hline Too high in carbohydrates for most people & $10(8)$ & $16.7(4)$ & $18.2(4)$ & - & - & - \\
\hline Too high in proteins for most people & $25(20)$ & - & $18.2(4)$ & $85.7(12)$ & $50(4)$ & - \\
\hline \multicolumn{7}{|c|}{ Which of the following is highest in carbohydrate? $p=0.372$} \\
\hline Baked chicken & $10(8)$ & - & $27.3(6)$ & $14.3(2)$ & - & - \\
\hline Swiss cheese & $15(12)$ & - & - & - & - & $100(12)$ \\
\hline Baked potato* & $45(36)$ & $66.7(16)$ & $54.5(12)$ & $57.1(8)$ & - & - \\
\hline Peanut butter & $30(24)$ & $33.3(8)$ & $18.2(4)$ & $28.6(4)$ & $100(8)$ & - \\
\hline \multicolumn{7}{|c|}{ Which of the following is highest in fat? $p=0.265$} \\
\hline *Low fat $(2 \%)$ milk & $80(64)$ & $100(24)$ & $81.8(18)$ & $42.9(6)$ & $50(4)$ & $100(12)$ \\
\hline Orange juice & - & - & - & - & - & - \\
\hline Corn & $20(16)$ & - & $18.2(4)$ & $57.1(8)$ & $50(4)$ & - \\
\hline Honey & - & - & - & - & - & - \\
\hline \multicolumn{7}{|c|}{ Which of the following is a "free food"? $p=0.496$} \\
\hline Any unsweetened food & $25(20)$ & $33.3(8)$ & $9.1(2)$ & $14.3(2)$ & $100(8)$ & - \\
\hline Any food that has "fat free" on the label & - & - & - & - & - & - \\
\hline Any food that has "sugar free" on the label & $30(24)$ & $16.7(4)$ & $36.4(8)$ & - & - & $100(12)$ \\
\hline Any food that has less than 20 calories per serving* & $45(36)$ & $50(12)$ & $54.5(12)$ & $85.7(12)$ & - & - \\
\hline
\end{tabular}

Table 2: Knowledge of nurses about the diet therapy.

\section{Blood Sugar Control}

The average level of knowledge about glycemic control was $60 \%$ i.e. medium knowledge (Table 3). Negative statistically significant correlation was determined $(p$
$0.036)$ between the question on the impact of physical activity and employment, as well as between questions about the treatment of low blood glucose levels and years of experience ( $\mathrm{p} \mathrm{0.002)}$ ).

\begin{tabular}{|l|c|c|c|c|c|c|c|}
\hline \multirow{2}{*}{} & \multirow{2}{*}{ Total \% (n) } & \multicolumn{5}{|c|}{ According to the years of work experience } \\
\cline { 3 - 7 } & & $\mathbf{0}$ & $\mathbf{5 - J a n}$ & $\mathbf{1 0 - J u n}$ & \multicolumn{1}{|c|}{$\mathbf{1 5 - N o v}$} & $\mathbf{1 6 - 2 0}$ \\
\hline HbA1C is a measure of average blood glucose level for the past: $\mathbf{p}=\mathbf{0 . 1 9 2}$ \\
\hline day & $10(8)$ & $16.7(4)$ & $18.2(4)$ & - & - & - \\
\hline week & - & - & - & - & - & - \\
\hline 6-12 weeks* & $65(52)$ & $66.7(16)$ & $63.6(14)$ & $42.9(6)$ & $50(4)$ & $100(12)$ \\
\hline
\end{tabular}




\section{Nursing \& Healthcare International Journal}

\begin{tabular}{|c|c|c|c|c|c|c|}
\hline 6 months & $25(20)$ & $16.7(4)$ & $18.2(4)$ & $57.1(8)$ & $50(4)$ & - \\
\hline \multicolumn{7}{|c|}{ Which is the best method for home glucose testing? $\mathrm{p}=0.000^{* *}$} \\
\hline Urine testing & - & - & - & - & - & - \\
\hline Blood testing* & $85(68)$ & $100(24)$ & $100(22)$ & $100(14)$ & $50(4)$ & $33.3(4)$ \\
\hline Both are equally good & $15(12)$ & - & - & - & $50(4)$ & $66.7(8)$ \\
\hline \multicolumn{7}{|c|}{ What effect does unsweetened fruit juice have on blood glucose $? \mathrm{p}=0.206$} \\
\hline Lowers it & - & - & - & - & - & - \\
\hline Raises it* & $65(52)$ & $66.7(16)$ & $63.6(14)$ & $14.3(2)$ & $100(8)$ & $100(12)$ \\
\hline Has no effect & $35(28)$ & $33.3(8)$ & $36.4(8)$ & $85.7(12)$ & - & - \\
\hline \multicolumn{7}{|c|}{ Which should not be used to treat a low blood glucose? $\mathrm{p}=-\mathbf{0 . 0 0 2}{ }^{* *}$} \\
\hline 3 hard candies & $20(16)$ & $16.7(4)$ & - & - & - & $100(12)$ \\
\hline $1 / 2$ cup orange juice & $10(8)$ & - & $18.2(4)$ & $28.6(4)$ & - & - \\
\hline 1 cup diet soft drink* & $40(32)$ & $66.7(16)$ & $27.3(6)$ & $14.3(2)$ & $100(8)$ & - \\
\hline 1 cup skim milk & $30(24)$ & $16.7(4)$ & $54.5(12)$ & $57.1(8)$ & - & - \\
\hline \multicolumn{7}{|c|}{ For a person in good control, what effect does exercise have on blood glucose? $p=0.420$} \\
\hline Lowers it* & $40(32)$ & $50(12)$ & $36.4(8)$ & - & - & $100(12)$ \\
\hline Raises it & $5(4)$ & $16.7(4)$ & 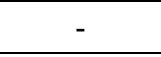 & - & - & - \\
\hline Has no effect & $55(44)$ & $33.3(8)$ & $63.6(14)$ & $100(14)$ & $100(8)$ & - \\
\hline \multicolumn{7}{|c|}{ What effect will an infection most likely have on blood glucose? $p=1.000$} \\
\hline Lowers it & $20(16)$ & $16.7(4)$ & $27.3(6)$ & $14.3(2)$ & $50(4)$ & - \\
\hline Raises it* & $60(48)$ & $50(12)$ & $72.7(16)$ & $28.6(4)$ & $50(4)$ & $100(12)$ \\
\hline Has no effect & $20(16)$ & $33.3(8)$ & - & $57.1(8)$ & - & - \\
\hline \multicolumn{7}{|c|}{ If a patient are sick with the flu, he/she should: $p=0.929$} \\
\hline Take less insulin & $5(4)$ & $16.7(4)$ & - & - & - & - \\
\hline Drink less liquids & - & - & - & - & - & \\
\hline Eat more proteins & $30(24)$ & - & $36.4(8)$ & $57.1(8)$ & - & $66.7(8)$ \\
\hline Test blood glucose more often* & $65(52)$ & $83.3(20)$ & $63.6(14)$ & $42.9(6)$ & $100(8)$ & $33.3(4)$ \\
\hline
\end{tabular}

Table 3. Knowledge of nurses on the control of blood sugar $\mathrm{p}^{* *}$ Statistically significant correlation at the level $\mathrm{p} \leq 0.01$

\section{The Effect of Insulin, and Possible Complications}

Control of blood sugar requires a certain level of knowledge about insulin therapy, which in this case is
75.7\%, which corresponds with a high level of knowledge (Table 4). There also appears to be a statistically significant correlation between knowledge of insulin therapy with the work experience $(\mathrm{p} \leq 0.05)$.

\begin{tabular}{|c|c|c|c|c|c|c|c|}
\hline \multirow{2}{*}{ Total \% (n) } & \multicolumn{5}{|c|}{ According to the years of work experience } \\
\cline { 3 - 7 } & & $\mathbf{0}$ & 5-Jan & 10-Jun & 15-Nov & $\mathbf{1 6 - 2 0}$ \\
\hline If a patient takes rapid-acting insulin, they are most likely to have a low blood glucose reaction in: p=0.759 \\
\hline Less than 2 hours* & $55(44)$ & $66.7(16)$ & $45.5(10)$ & $100(14)$ & $50(4)$ & - \\
\hline 3-5 hours & $25(20)$ & $16.7(4)$ & - & - & $50(4)$ & $100(12)$ \\
\hline 6-12 hours & $20(16)$ & $16.7(4)$ & $54.5(12)$ & - & - & - \\
\hline More than 13 hours & - & - & - & - & - & - \\
\hline
\end{tabular}




\begin{tabular}{|c|c|c|c|c|c|c|}
\hline \multicolumn{7}{|c|}{ If a patient takes rapid-acting insulin, they are most likely to have a low blood glucose reaction in: $p=0.759$} \\
\hline Less than 2 hours* & $55(44)$ & $66.7(16)$ & $45.5(10)$ & $100(14)$ & $50(4)$ & - \\
\hline 3-5 hours & $25(20)$ & $16.7(4)$ & - & - & $50(4)$ & $100(12)$ \\
\hline 6-12 hours & $20(16)$ & $16.7(4)$ & $54.5(12)$ & - & - & - \\
\hline More than 13 hours & - & - & - & - & - & - \\
\hline \multicolumn{7}{|c|}{$\begin{array}{l}\text { Patient realizes just before lunch that they forgot to take their insulin at breakfast. What should they do now? } \\
p=0.064\end{array}$} \\
\hline Skip lunch to lower blood glucose & - & - & - & - & - & - \\
\hline \begin{tabular}{|c|} 
Take the insulin that they usually take \\
at breakfast
\end{tabular} & $10(8)$ & $16.7(4)$ & $18.2(4)$ & - & - & - \\
\hline \begin{tabular}{|c|}
$\begin{array}{c}\text { Take twice as much insulin as they } \\
\text { usually take at breakfast }\end{array}$ \\
\end{tabular} & $5(4)$ & - & $18.2(4)$ & - & - & - \\
\hline \begin{tabular}{|c|}
$\begin{array}{c}\text { Check blood glucose levels to decide } \\
\text { how much insulin to take* }\end{array}$ \\
\end{tabular} & $85(68)$ & $83.3(20)$ & $63.6(14)$ & $100(14)$ & $100(8)$ & $100(12)$ \\
\hline \multicolumn{7}{|c|}{ When patients are beginning to have a low blood glucose reaction, they should: $p=0.099$} \\
\hline Exercise & - & - & - & - & - & - \\
\hline Lie down and rest & $10(8)$ & $16.7(4)$ & $18.2(4)$ & - & - & - \\
\hline Drink some juice * & $90(72)$ & $83.3(20)$ & $81.8(18)$ & $100(14)$ & $100(8)$ & $100(12)$ \\
\hline Take rapid-acting insulin & - & - & - & - & - & - \\
\hline \multicolumn{7}{|c|}{ A low blood glucose reaction may be caused by: $p=0.183$} \\
\hline Too much insulin* & $75(60)$ & $83.3(20)$ & $63.6(14)$ & $42.9(6)$ & $100(8)$ & $100(12)$ \\
\hline Too little insulin & $15(12)$ & - & $18.2(4)$ & $57.1(8)$ & - & - \\
\hline Too much food & $10(8)$ & $16.7(4)$ & $18.2(4)$ & - & - & - \\
\hline Too little exercise & - & - & - & - & - & - \\
\hline \multicolumn{7}{|c|}{ If patient take their morning insulin but skip breakfast, the blood glucose levels will usually: $p=0.030^{*}$} \\
\hline Increase & $20(16)$ & $33.3(8$ & $36.4(8)$ & - & - & - \\
\hline Decrease* & $80(64)$ & $66.7(16)$ & $63.6(14)$ & $100(14)$ & $100(8)$ & $100(12)$ \\
\hline Remain the same & - & - & - & - & - & - \\
\hline \multicolumn{7}{|c|}{ High blood glucose may be caused by: $p=0.853$} \\
\hline Not enough insulin* & $85(68)$ & $83.3(20)$ & $100(22)$ & $42.9(6)$ & $100(8)$ & $100(12)$ \\
\hline Skipping meals & $15(12)$ & $16.7(4)$ & - & $57.1(8)$ & - & - \\
\hline Delaying your snack & - & - & - & - & - & - \\
\hline Skipping your exercise & - & - & - & - & - & - \\
\hline \multicolumn{7}{|c|}{ A low blood glucose reaction may be caused by: $p=0.165$} \\
\hline Heavy exercise* & $60(48)$ & $66.7(16)$ & $27.3(6)$ & $42.9(6)$ & $100(8)$ & $100(12)$ \\
\hline Infection & $15(12)$ & $16.7(4)$ & $36.4(8)$ & - & - & - \\
\hline Overeating & - & - & - & - & - & - \\
\hline Not taking your insulin & $25(20)$ & $16.7(4)$ & $36.4(8)$ & $57.1(8)$ & - & - \\
\hline
\end{tabular}

Table 4: Knowledge of nurses about insulin therapy.

$\mathrm{p}^{*}$ Statistically significant correlation at the level of $\mathrm{p} \leq 0.05$.

\section{Chronic Complications of Diabetes}

In this part the respondents have achieved 63\% correct answers, which correspond to the middle level of knowledge (Table 5). It did not show a statistically significant correlation between employment or years of service. 


\section{Nursing \& Healthcare International Journal}

\begin{tabular}{|c|c|c|c|c|c|c|}
\hline & \multirow{2}{*}{ Total \% (n) } & \multicolumn{5}{|c|}{ According to the years of work experience } \\
\hline & & $\mathbf{0}$ & 5-Jan & 10-Jun & 15-Nov & 16-20 \\
\hline \multicolumn{7}{|c|}{ The best way to take care of feet is to: $p=0.761$} \\
\hline Look at them and wash them each day* & $45(36)$ & $50(12)$ & $45.5(10)$ & $71.4(10)$ & - & $33.3(4)$ \\
\hline Massage them with alcohol each day & $45(36)$ & $33.3(8)$ & $36.4(8)$ & $28.6(4)$ & $100(8)$ & $66.7(8)$ \\
\hline Soak them for one hour each day & $10(8)$ & $16.7(4)$ & $18.2(4)$ & - & - & - \\
\hline Buy shoes a size larger than usual & - & - & - & - & - & - \\
\hline \multicolumn{7}{|c|}{ Eating foods lower in fat decreases risk for: $p=0.757$} \\
\hline Nerve disease & $15(12)$ & $16.7(4)$ & $18.2(4)$ & - & $50(4)$ & - \\
\hline Kidney disease & - & - & - & - & - & \\
\hline Heart disease * & $85(68)$ & $83.3(20$ & $81.8(18)$ & $100(14)$ & $50(4)$ & $100(12)$ \\
\hline Eye disease & - & - & - & - & - & - \\
\hline \multicolumn{7}{|c|}{ Numbness and tingling may be symptoms of: $p=0.555$} \\
\hline Kidney disease & $10(8)$ & $16.7(4)$ & - & $28.6(4)$ & - & - \\
\hline Nerve disease * & $90(72)$ & $83.3(20)$ & $100(22)$ & $71.4(10)$ & $100(8)$ & $100(12)$ \\
\hline Eye disease & - & - & & - & - & - \\
\hline Liver disease & - & - & - & - & - & - \\
\hline \multicolumn{7}{|c|}{ Which of the following is usually not associated with diabetes: $p=0.610$} \\
\hline Vision problems & $5(4)$ & - & - & $28.6(2)$ & - & - \\
\hline Kidney problems & $5(4)$ & - & $18.2(4)$ & - & - & - \\
\hline Nerve problems & $5(4)$ & $16.7(4)$ & - & - & - & - \\
\hline Lung problems* & $85(68)$ & $83.3(20)$ & $81.8(18)$ & $71.4(10)$ & $100(8)$ & $100(12)$ \\
\hline \multicolumn{7}{|c|}{ Signs of ketoacidosis (DKA) include: $p=0.871$} \\
\hline Shakiness & $10(8)$ & - & $18.2(4)$ & - & - & $33.3(4)$ \\
\hline Sweating & $35(28)$ & $50(12)$ & $18.2(4)$ & $57.1(8)$ & - & $33.3(4)$ \\
\hline Vomiting * & $10(8)$ & - & $18.2(4)$ & $28.6(4)$ & - & - \\
\hline Low blood glucose & $45(36)$ & $50(12)$ & $45.5(10)$ & $14.3(2)$ & $100(8)$ & $33.3(4)$ \\
\hline
\end{tabular}

Table 5: Knowledge of nurses on the management of chronic complications.

\section{Discussion}

Respondents have low knowledge (58.75\%) on diet in diabetics, medium expertise $(60 \%)$ on the control of blood sugar, and high skills (75.7\%) of insulin therapy and hypoglycemia and medium knowledge (63\%) of chronic complications.

Considering employment and seniority, nurses with several years of experience are better informed on the control of blood sugar and insulin therapy ( $\mathrm{p} \leq 0.05)$. Nurses in their daily work are regularly confronted with such patients, and with direct work they can acquire the necessary information, and transfer it to other newly discovered patients. Also, higher level of knowledge in the field of chronic complications (foot care, signs of ketoacidosis) achieved people with 6-10 years of experience. However younger, unemployed, students have greater knowledge about nutritional principles.

In the field of nutrition, the reverse correlation between employment and knowledge has been identified ( $p=-0.013$ ), it is observed that a higher percentage of correct answers was among students with fewer years of experience, which can be explained by the relative fresh knowledge they got during secondary medical school. While employees with over 10 years of experience obviously have forgotten the principles of proper nutrition in diabetic patients, and are not able to consult in this area. 


\section{Nursing \& Healthcare International Journal}

We can say that the students who are unemployed have better theoretical knowledge while better practical skills are present by employed students on the basis of which we accept this hypothesis. If we look at all these segments as a whole, the respondents completed the test of knowledge about diabetes with $64.4 \%$ accuracy, which according to the criteria described above corresponds to the middle level of knowledge about diabetes.

It warns us that we have to organize seminars and educational programs on the subject of diabetes management for refreshment and for the expansion of knowledge of nurses. Since half of the respondents is conducting regular training of diabetic patients, there is a possibility of transferring of inaccurate and incomplete information. Respondents have very low knowledge about nutrition and the composition of carbohydrate foods. However diabetes primarily (and very often exclusively) is treated with a diet, with patient education necessary for proper selection and food preparation.

The level of knowledge about chronic complications is very concerning, primarily at questions in diabetic foot care and in recognizing signs of ketoacidosis. Chronic complications of diabetes create the greatest burden for the health sector, their occurrence can be prevented and slowed down by adequate health educational work and successful self-care. We are obligated to teach patients how to take care of their feet, so that they recognize the signs of high and low blood sugar and what to do in these cases. We have to motivate them for continuous medical monitoring to detect possible complications.

Education for self-management of diabetes is an important component in improving the state of the patients and in preventing the complications associated with diabetes. Every person who has diabetes is responsible for controlling this condition and therefore must understand the basic principles of pathophysiology and of the disease's treatment so that it would be more efficient. Clearly, the importance of preventive measures is high. Investing in relatively simple and inexpensive procedures leads to a significant positive effect on human health and therefore reduces the cost of their treatment [12].

Knowledge deficits of diabetes care represent a major risk for delivery of unsafe practice. Significant knowledge deficiencies are demonstrated in core aspects of diabetes care amongst nurses, and barriers to nurses' diabetes knowledge acquisition are also identified. Research on the registered and practice nurse in Canada shows the highest level of knowledge in the field of nutrition and the management of hypo and hyperglycemia, and the lowest level of knowledge about self-management of diabetes, oral medicines and insulin. However, most clinical nurses consider that there is a need for additional education [13]. Also, in West Pennsylvania as the main cause of low level of knowledge and a moderate level of self-efficacy of nurses, they emphasize that nurses are not trained for counseling and education, and that they are not familiar with techniques of increasing self-confidence [14].

In West Africa, similar problems have been discovered, the knowledge of nursing medics about nutritional management of diabetes is low, which calls into question the effectiveness of the nurse counseling. Almost $90 \%$ of nurses do not know the recommended daily intake of carbohydrates for diabetic patients, and over $70 \%$ of nurses consider that diabetic patients can exclude any of the major nutrients from their meals. A higher level of knowledge was discovered among the meds who attended a course on nutrition and counseling for diabetes patients during and after school [15].

In Saudi Arabia the nurses generally had a positive view of their diabetes knowledge, with a mean score of 46.9 (of maximum 60) for the Diabetes Self-Report Tool, but their actual knowledge scores ranged with a mean score of 25.4 (of maximum of 49). So, findings indicated a significant gap between participants' perceived and actual knowledge [16]. All in all, findings indicate widespread serious and persistent deficiencies in the knowledge of nurses in many aspects of diabetes care and about diabetes, and urgent strategies are needed to overcome identified obstacles to acquire knowledge [17].

Study on the education of clinical nurses on diabetes conducted in South Korea and India. After conducting several workshops on diabetes, it showed not only a higher level of knowledge about diabetes, but also the practical application of knowledge. In the post test more than $50 \%$ of the subjects achieved results between 91 $100 \%$, and about half between $71-90 \%$, therefore, the nurses had greater confidence in providing health education after attending the program [18].

These findings call for the need to introduce educational programs. Therefore, to enable our health care system to implement educational programs and to boast with such results, it is necessary first of all to invest in the training of senior nurses for the implementation of such programs. 


\section{Conclusion}

Highly educated nurses are responsible for patient education to successfully manage diabetes. In this study the average student nurses' knowledge about diabetes was $64.4 \%$, which corresponds to the middle level of knowledge about diabetes. The lowest level of knowledge demonstrated was about nutrition. In order to ensure the transmission of quality information for diabetics, it is necessary to update the knowledge of health workers, including the latest information on diabetes, in the form of annual seminars. It is not enough to just listen to new information, but they must be implemented in everyday practices.

\section{Highlights}

- The basic form of diabetes treatment is a change in the life style, especially in the field of nutrition.

- Nurses are responsible for training and education of patients with diabetes.

- Diabetes self-management depends primarily on the knowledge and ability of the nurse to transfer information. However, in the education of nurses, we face low knowledge of adequate nutrition in diabetes.

- For successful education in diabetes, we need to find an adequate solution for nursing training.

Authors' declaration

For the manuscript of "Diabetes and education -Knowledge of high school nursing students about the management of diabetes mellitus" under the authorship of Hajnalka Požar, Jovanka Novaković-Paro and Dragana Simin.

We give the statement:

-that the work is original and has not been previously published elsewhere (either partially or totally), and is not in the process of being considered for publication in another journal.

-the manuscript publication is approved by all authors and by the responsible authorities where the work was carried out,

-and that, if accepted, it will not be published elsewhere in the same form, in English or in any other language, including electronically without the written consent of the copyright-holder. - that all authors meet the criteria for authorship, have approved the final article and that all those entitled to authorship are listed as authors.

The authors equaly participated in the preparation of the manuscript. P.H. participated in the conception and design of the study, in the acquisition, analysis and interpretation of data, in statistical analysis and drafting the article. J.N.P. and D.S. participated in the conception and design of the study, in the interpretation of data and in critically revising it for important intellectual content. The final version of the article has been read and approved by all of the authors.

-The importance of this work is reflected in the assessment of knowledge of nurses about health education work with diabetes. The novelty is, that this topic was first conducted in our country, making recommendations for improving the education of nurses.

Ethical approval: To conduct research, the written consent of the school director was obtained. This research did not receive financial support or any specific grant from funding agencies in the public, commercial, or not-for-profit sectors.

Conflict of interest (financial, personal or other): None.

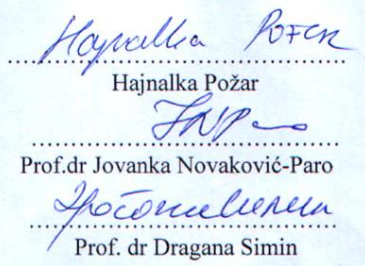

Požar H, et al. Diabetes and Education - Knowledge Level about Diabetes 


\section{Nursing \& Healthcare International Journal}

\section{References}

1. WHO (2013) Global action plan for the prevention and control of noncommunicable diseases 2013-2020, World Health Organization, pp: 1-103.

2. Sicree R, Shaw JR, Zimmet PZ (2009) The Global Burden of diabetes. In: Gan D (Eds.) Diabetes Atlas. $4^{\text {th }}$ (Edn.), Brussels: International Diabetes Federation.

3. Register for diabetes in Serbia (2014) The incidence of and mortality from diabetes in Serbia, 2014. Report no. 9. Belgrade: Institute of Public Health of Serbia. Dr Milan Jovanović Batut.

4. (2014) The results of studies on health population in Serbia 2013. Belgrade: Ministry of Health of the Republic of Serbia and the Institute of Public Health of Serbia. Dr Milan Jovanovic Batut.

5. Diabetes Control and Complications Trial Research Group, Nathan DM, Genuth S, Lachin J, Cleary P, et al. (1993) The effect of intensive treatment of diabetes on the development of long term complications in insulin dependent diabetes mellitus. $\mathrm{N}$ Engl J Med 329(14): 977-986.

6. Weinberger M, Kirkman MS, Samsa GP, Shortliffe EA, Landsman PB (1995) A nurse-coordinated intervention for primary care patients with noninsulin dependent diabetes mellitus: Impact on glycemic control and health related quality of life. J Gen Intern Med 10(2): 59-66.

7. Kaur S, Walia I (2007) Knowledge of diabetes mellitus amongst nursing students - Effect of an intervention. Nursing and Midwifery Research Journal 3(1): 30-35.

8. Drass JA, Muir Nash J, Boykin PC, Turek JM, Baker KL (1989) Perceived and actual level of knowledge of diabetes mellitus among nurses. Diabetes Care 12: 351-356.

9. Michigan Diabetes Research Center (2016) Michigan (America): University of Michigan Health System. Tools for Health Professionals-Survey Instruments.

10. Kassahun T, Gesesew H, Mwanri L, Eshetie T (2016) Diabetes related knowledge, self-care behaviours and adherence to medications among diabetic patients in Southwest Ethiopia: a cross-sectional survey. BMC Endocr Disord 16(1): 28.

11. Fitzgerald JT, Funnell MM, Hess GE, Barr PA, Anderson RM, et al. (1998) The reliability and validity of a brief diabetes knowledge test. Diabetes Care 21(5): 706-710.

12. Powers MA, Bardsley J, Cypress M, Duker P, Funnell MM, et al. (2015) Diabetes Self-management Education and Support in Type 2 Diabetes. A Joint Position Statement of the American Diabetes Association, the American Association of Diabetes Educators, and the Academy of Nutrition and Dietetics. Diabetes Educ 20(10): 1-14.

13. Vincent C, Hall P, Ebsary S, Hannay S, Hayes Cardinal L, et al. (2016) Knowledge Confidence and Desire for Further Diabetes-Management Education among Nurses and Personal Support Workers in Long-Term Care. Can J Diabetes 40(3): 226-323.

14. Devido JA, Doswell WM, Braxter BJ, Spatz DL, Dorman JS, et al. (2017) Experiences of Parish Nurses in Providing Diabetes Education and Preconception Counseling to Women With Diabetes. J Obstet Gynecol Neonatal Nurs 46(2): 248-257.

15. Mogre V, Ansah GA, Marfo DN, Garti HA (2015) Assessing nurses' knowledge levels in the nutritional management of diabetes. International Journal of Africa Nursing Sciences 3: 40-43.

16. Alotaibi A, Gholizadeh L, Al-Ganmi A, Perry L (2017) Examining perceived and actual diabetes knowledge among nurses working in a tertiary hospital. Applied Nursing Research 35: 24-29.

17. Alotaibi A, Al Ganmi A, Gholizadeh L, Perry L (2016) Diabetes knowledge of nurses in different countries: An integrative review. Nurse Educ Today 39: 32-49.

18. Young Hee K (2015) A study of Clinical Nurses' knowledge about Critical Practice Guideline for Diabetes Mellitus. Advanced Science and Technology Letters (Healthcare and Nursing) 88: 130-134. 